\title{
Locare, allocare e allocazione
}

\section{Claudio Giovanardi}

PUBBLICATO: 9 APRILE 2021

\section{Quesito:}

Sono arrivati al nostro servizio di consulenza alcuni quesiti riguardanti il significato dei verbi locare e allocare e l'esistenza della variante allogare. Altre domande invece riguardano il termine allocamento e il suo rapporto con allocazione.

\section{Locare, allocare e allocazione}

$\mathrm{N}$ umerosi quesiti riguardano i verbi locare e allocare e altri vocaboli della stessa famiglia. Procediamo dunque con ordine. Il verbo locare deriva dal latino locāre, a sua volta da lócus 'luogo', e presenta due significati principali: il primo, ricco di attestazioni letterarie, ma ormai in disuso, è quello di 'collocare, mettere in un luogo'; il secondo, invece attualissimo, equivale a 'affittare, dare in locazione': locare un immobile, un appartamento, un bene. La fortuna di questa seconda accezione è dovuta a una duplice ragione: da un lato l'uso che se ne fa nel linguaggio giuridico e burocratico, dall'altro il fatto che si tratta di un regionalismo diffuso in diverse aree dell'Italia centromeridionale nelle quali viene preferito ad affittare. Le stesse considerazioni valgono anche per il participio passato in funzione aggettivale locato, ormai desueto nel significato di 'collocato, ubicato', mentre è diffuso col valore di 'affittato', anche in questo caso con duplice azione del linguaggio giuridico e degli usi regionali. Il valore spaziale si mantiene ancora nella forma altolocato, letteralmente 'collocato in alto', che però oggi può essere usata solo in riferimento al rango sociale di una persona.

Per quanto riguarda il verbo allocare, che viene dal latino tardo allocāre, si tratta di una variante formale, considerata desueta, di allogare (in cui si ha la sonorizzazione della velare sorda, esattamente come avviene in luogo a partire da lócum). Il verbo allogare, oggi certamente poco usato, significa 'mettere, collocare in un luogo' e può riferirsi tanto a cose quanto a persone (anche se in quest'ultimo caso sarebbe preferibile usare alternative come assegnare o destinare). Nel linguaggio economico, invece, la forma allocare (e non, in questo caso, allogare) si è diffusa e ha assunto il significato tecnico di 'ripartire, distribuire': allocare le risorse in piu uffici, allocare le entrate nelle poste di bilancio; il verbo, transitivo, è normalmente seguito dalla proposizione in, che indica il "luogo" (materiale o virtuale) dove qualcosa viene allocato per un determinato fine. Probabilmente allocare nel linguaggio dell'economia si è rideterminato semanticamente a partire dal termine allocazione (a sua volta condizionato dal francese allocation e dall'inglese allocation), il cui significato è 'ripartizione di beni e servizi fra più enti o più soggetti', e che non può essere sostituito da *allocamento.

\section{Cita come:}

Claudio Giovanardi, Locare, allocare e allocazione , "Italiano digitale", XVII, 2021/2 (aprile-giugno)

DOI: $10.35948 / 2532-9006 / 2021.6515$

Copyright 2021 Accademia della Crusca

Pubblicato con licenza creative commons CC BY-NC-ND 